\title{
Cost minimization of repairable systems subject to availability constraints using efficient cuckoo optimization algorithm
}

\author{
Mohamed Arezki Mellal $^{1,2}$ ( ) | Enrico Zio ${ }^{3,4}$ | Edward J. Williams ${ }^{5,6}$
}

\author{
${ }^{1}$ LMSS, Faculty of Technology, M'Hamed \\ Bougara University, Boumerdes, Algeria \\ ${ }^{2}$ Center for Advanced Life Cycle \\ Engineering (CALCE), University of \\ Maryland, College Park, Maryland, USA \\ ${ }^{3}$ CRC, Mines ParisTech, PSL Research \\ University, Paris, France \\ ${ }^{4}$ Energy Department, Politecnico di \\ Milano, Milano, Italy \\ ${ }^{5}$ Industrial and Manufacturing Systems \\ Engineering Department, College of \\ Engineering and Computer Science, \\ University of Michigan, Dearborn, \\ Michigan, USA \\ ${ }^{6}$ Decision Sciences, College of Business, \\ University of Michigan, Dearborn, \\ Michigan, USA

\section{Correspondence} \\ Mohamed Arezki Mellal, Center for \\ Advanced Life Cycle Engineering \\ (CALCE), University of Maryland, College \\ Park, Maryland, USA. \\ Email: mellal.mohamed@univ- \\ boumerdes.dz; mellal.mohamed@gmail. \\ com; mamellal@umd.edu
}

\begin{abstract}
System availability is a key element for any industry. System designers and operators try to do their best to maintain the required availability of the systems to avoid production stoppages. They set up and undertake different maintenances, and these interventions imply cost. Therefore, the goal is to minimize the cost, but considering the constraint of the availability requirement. The problem involves three main aspects: redundancy allocation, component failure rates, and repair rates. In this paper, a novel solution approach is proposed based on an efficient cuckoo optimization algorithm (EF-COA). Two numerical case studies are solved, and the results confirm the effectiveness of the approach proposed.
\end{abstract}

\section{K E Y W O R D S}

availability requirement, efficient cuckoo optimization algorithm (EF-COA), repairable systems, system cost

\section{1 | INTRODUCTION}

A competitive industrial plant or infrastructure requires a highly dependable system with minimum functioning cost. The system dependability is a challenge that simultaneously incorporates reliability, availability, maintainability, and safety (RAMS). ${ }^{1}$ The focus of the designer depends on the target, criteria, and system nature, such as nuclear power plants $^{2,3}$ and network systems (eg, electric power transmission/distribution systems, water/oil/gas distribution systems, computer/communication systems, and rail/road transportation systems). ${ }^{4}$

Higher RAMS allocation improves system dependability but also increases system cost. $^{5}$ Most of RAMS problems are described as optimization problems with single or multi objective functions subject to the constraints fixed by the specifications (eg, weight and volume). Evolutionary computation methods, also referred to artificial intelligence methods (AI), have successfully dealt with RAMS problems. In previous studies, ${ }^{6-13}$ the authors used the artificial bee colony $(\mathrm{ABC})^{6}$, immune based algorithm (IA) ${ }^{7}$, differential evolution with Lévy flight $(\mathrm{DE})^{8}$, the biogeography-based optimization algorithm $(\mathrm{BBO})^{9}$, particle swarm optimization (PSO) $)^{10,11}$, penalty guided stochastic fractal search ${ }^{12}$, and the gray wolf optimizer algorithm ${ }^{13}$ for system reliability models. In Marseguerra et al, ${ }^{1}$ a multi-objective approach 
based on genetic algorithm has been presented for simultaneously dealing with the following objectives: system reliability, system maintainability, system safety, and cost (RAMS\&C). In Liu, ${ }^{14,15}$ a method was proposed combining Tabu search and genetic algorithm (TA-GA) for minimizing the system cost under availability constraint. An ant algorithm for single and multi-objective system reliability problem has been developed in Shelokar et al. ${ }^{16}$ Recently, three evolutionary computation methods have been applied to a pharmaceutical plant in order to increase the overall system reliability ${ }^{17}$. The maintainability of a system by considering the failure and repair processes has been investigated in Cha and Finkelstein, ${ }^{18}$ whereas a new mathematical model of reliability for multi-state degraded repairable system has been proposed in Yu et al. ${ }^{19}$

The great challenge is to effectively deal with the dependability of the system and improve its elements. In this paper, we propose a novel solution approach for minimizing the system cost under system availability constraints, by resorting to a modification of the basic cuckoo optimization algorithm $(\mathrm{COA})^{20}$, in the present work called efficient cuckoo optimization algorithm (EF-COA). The remainder of the paper is organized as follows. Section 2 describes the system cost minimization problem subject to availability constraint. Section 3 presents the schemes of the EF-COA. In Section 4, two numerical case studies are presented. Finally, conclusions are drawn at closure.

\section{2 | PROBLEM DESCRIPTION}

The general mathematical formulation of the considered cost minimization problem of repairable systems is given as follows $^{14,15}$ :

$$
\text { Minimize } C_{S}(n, \lambda, \mu)=C_{S}\left(n_{1}, n_{2}, \ldots, n_{m} ; \lambda_{1}, \lambda_{2}, \ldots, \lambda_{m} ; \mu_{1}, \mu_{2}, \ldots, \mu_{m}\right)
$$

where $C_{S}(\bullet)$ is the total system cost, $n_{i}$ is the number of redundant components in the $i$ th subsystem, $\lambda_{i}$ is the failure rate of the components in the $i$ th subsystem, and $\mu_{i}$ is the repair rate of the components in the $i$ th subsystem,

subject to

$$
\begin{aligned}
& g_{j}\left(n_{1}, n_{2}, \ldots, n_{m}\right) \leq b, \\
& A_{S}(n, \lambda, \mu) \geq A_{S}^{*}, \\
& n_{i} \geq 1 ; n_{i} \in \mathbb{Z}^{+} \\
& \lambda_{i} \in\left[\lambda_{i L}, \lambda_{i U}\right] \subset \mathfrak{R}^{+} \\
& \mu_{i} \in\left[\mu_{i L}, \mu_{i U}\right] \subset \mathfrak{R}^{+}, \\
& i=1,2, \ldots, m,
\end{aligned}
$$

where $g(\bullet)$ is the set of constraints, $b$ is the vector of resource limitation, $A_{S}(\bullet)$ is the system availability, $A_{S}^{*}$ is the system availability requirement, and $m$ is the number of subsystems in the system.

\section{I EFFICIENT COA}

The COA is a bio-inspired evolutionary optimization method developed by Rajabioun. ${ }^{20}$ The basic principles are based on the lifestyle and behavior of the birds, cuckoos, for their reproduction. Several works available in the literature used the main concepts of this algorithm for solving various engineering problems, such as multivariable controller design ${ }^{20}$, replacement of obsolete components in industrial plants ${ }^{21,22}$, data clustering ${ }^{23}$, machining parameters ${ }^{24-26}$, job scheduling $^{27}$, warranty period definition ${ }^{28}$, nonconvex combined heat and power economic dispatch ${ }^{29}$, and recognition of control chart patterns ${ }^{30}$. The standard COA implies the major steps reported in Appendix A. ${ }^{20}$

To effectively solve the system cost minimization subject to the availability constraint described in Section 2, the basic COA is improved for better performance and the new approach is called EF-COA. The new steps of the algorithm are described as follows: 
Step 1. Random initialization of nests.

A fixed number of habitats and one cuckoo for each habitat only are considered. A random number of nests is generated for each habitat separately. Each nest represents a potential solution as follows:

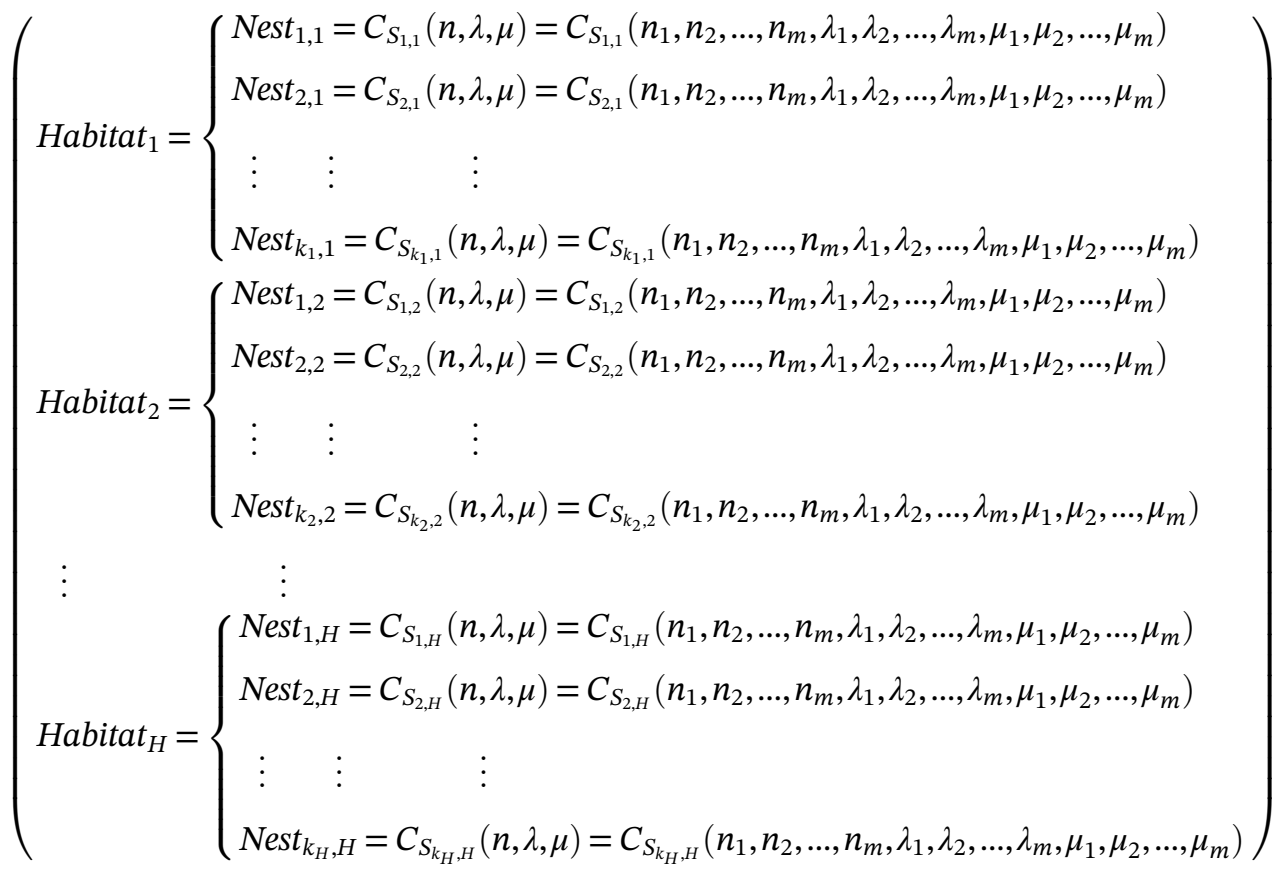

$$
\begin{aligned}
& k_{1}, k_{2}, \ldots, k_{H} \in\{2,3,4, \ldots, K\},
\end{aligned}
$$

where $H$ is the number of habitats and $K$ is the maximum number of nests which can be generated in the habitats.

Step 2. Evaluate the potential solutions.

The objective function value of each nest in each habitat is evaluated as follows:

$$
\operatorname{Nest}(n, \lambda, \mu)=C_{S}(n, \lambda, \mu)=C_{S}\left(n_{1}, n_{2}, \ldots, n_{m}, \lambda_{1}, \lambda_{2}, \ldots, \lambda_{m}, \mu_{1}, \mu_{2}, \ldots, \mu_{m}\right)
$$

Step 3. Constraint handling.

To deal with the inequality constraint described in Equations (2) and (3), the penalty method is used and the constrained problem is converted to an unconstrained one by adding penalty terms as follows ${ }^{29,31,32}$ :

$$
\operatorname{NEST}(n, \lambda, \mu)=\operatorname{Nest}(n, \lambda, \mu)+\Omega\left\langle A_{S}(n, \lambda, \mu)\right\rangle+\Phi_{j} \sum_{j=1}^{M}\left\langle g_{j}(n)\right\rangle,
$$

where $\operatorname{NEST}(n, \lambda, \mu)$ is the fitness value, $\operatorname{Nest}(n, \lambda, \mu)$ is the objective function value, $A_{S}(n, \lambda, \mu)$ is the system availability constraint, $g_{j}(n)$ are the other inequality constraints, $M$ is the number of constraints, and $\Omega$ and $\Phi$ are penalty parameters. The values of these parameters are set by trial-and-error and based on experience. The operator $\langle\cdot\rangle$ denotes the absolute value of the operand if it is negative; otherwise, it is zero. The real numbers of the vector of redundancy allocation $n$ are rounded to the nearest integer value.

Step 4. Identification of the best solution (minimum cost) and migration. 
All the nests of each habitat are classified and the best one is identified. The worst nests in each habitat mean that the eggs were recognized by the host birds and have been destroyed. Therefore, the best habitat includes the identified best nest (minimum system cost) and implies that this habitat represents the migration target for the cuckoo as follows:

$$
\text { Habitat }_{\text {Best }}=\left\{\text { Nest }_{\text {Best }}\right\}=C_{S_{\text {Best }}}(n, \lambda, \mu)=C_{S_{\text {Best }}}\left(n_{1}, n_{2}, \ldots, n_{m}, \lambda_{1}, \lambda_{2}, \ldots, \lambda_{m}, \mu_{1}, \mu_{2}, \ldots, \mu_{m}\right),
$$

where Habitat ${ }_{\text {Best }}$ is the best habitat with the best Nest $t_{\text {Best }}$.

Step 5. Use the best solution of the last previous cuckoo's generation (iteration) in the next one.

The best nest (best solution) of the last previous cuckoo's generation (ie, iteration) is considered a fixed nest for each habitat in the current iteration and the remaining nests are randomly generated. This step improves the solution's quality from one iteration to the next as follows:

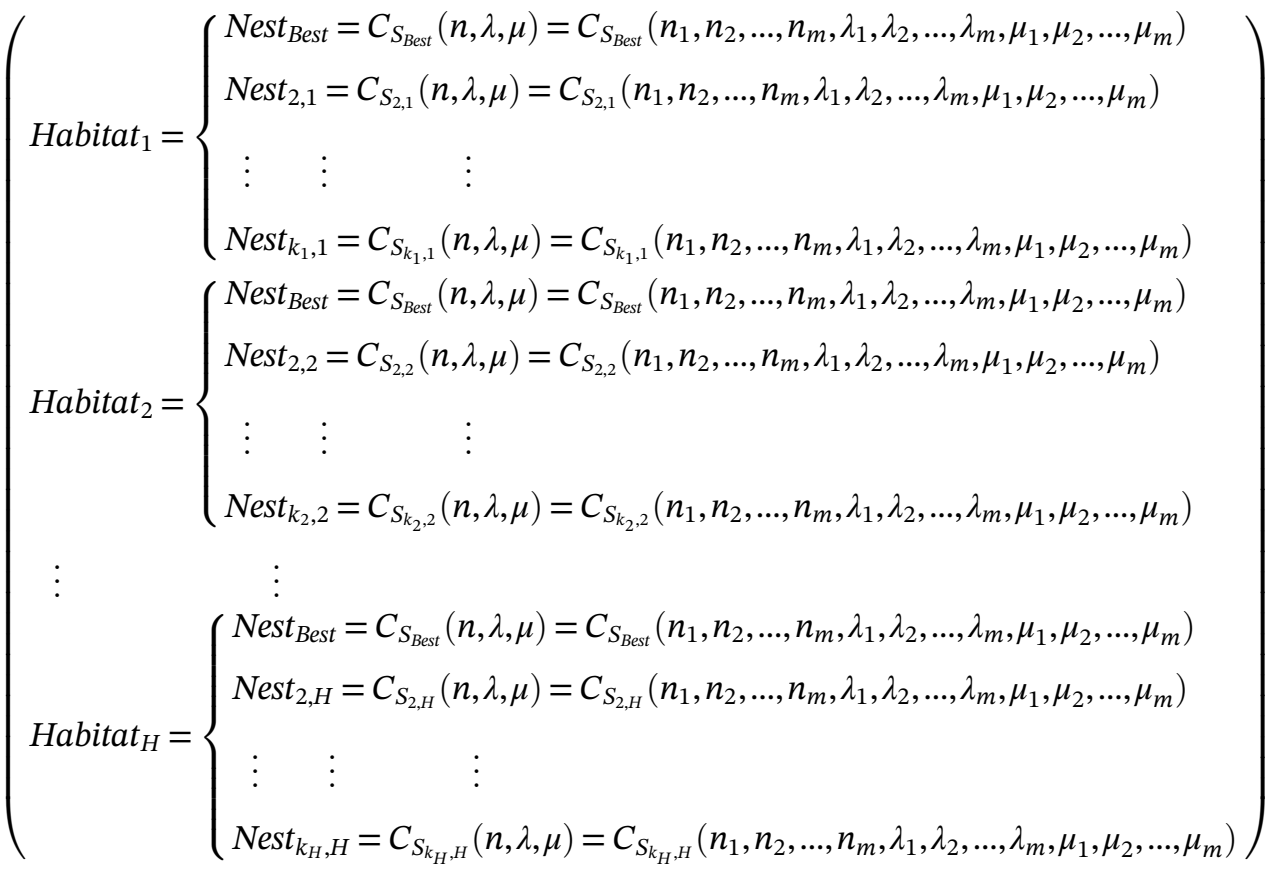

$$
\begin{aligned}
& k_{1}, k_{2}, \ldots, k_{H} \in\{2,3,4, \ldots, K\}
\end{aligned}
$$

Step 6. Steps 2 to 5 are repeated for a fixed number of iterations; then, the minimum system cost with the optimal values are displayed.

The pseudo-code of the developed EF-COA for the cost minimization of repairable systems subject to availability constraint is presented in Algorithm 1, and Figure 1 shows its flowchart.

\section{4 | CASE STUDIES}

\section{1 | Parallel-series system}

The overall system cost of five subsystems connected in parallel-series configuration (see Figure 2) is given by the mathematical model ${ }^{14}$ as follows: 


$$
\text { Minimize } C_{s}(n, \lambda, \mu)=\sum_{i=1}^{5}\left[\left(\alpha_{i}\left(\lambda_{i}\right)^{-\beta_{i}}+\mu_{i} m c_{i}\right)\left(n_{i}+\exp \left(n_{i} / 4\right)\right)\right]
$$

Subject to

$$
\begin{gathered}
\sum_{i=1}^{5} p_{i}\left(n_{i}\right)^{2} \leq 150, \\
\sum_{i=1}^{5} w_{i} n_{i} \exp \left(n_{i} / 4\right) \leq 200, \\
\prod_{i=1}^{5}\left[1-\left(1-\frac{\mu_{i}}{\lambda_{i}+\mu_{i}}\right)^{n_{i}}\right] \geq 0.9, \\
n_{i} \geq 1 ; n_{i} \in \mathbb{Z}^{+} \\
\lambda_{i} \in\left[10^{-7}, 10^{-3}\right] \subset \mathfrak{R}^{+} \\
\mu_{i} \in\left[32 \times 10^{-7}, 32 \times 10^{-3}\right] \subset \mathfrak{R}^{+} \\
i=1,2, \ldots, 5,
\end{gathered}
$$

where Equation (10) is the system design configuration constraint of weight, Equation (11) is the system design configuration constraint of the product of weight and volume, and Equation (12) is the system availability requirement constraint. The above problem involves five integer variables and 10 real variables. Table 1 reports the relevant data.

\section{2 | n-stage standby system}

The $n$-stage standby system considered includes five subsystems ${ }^{15}$ (see Figure 3), and the corresponding optimization reads:

$$
\text { Minimize } C_{s}(n, \lambda, \mu)=\sum_{i=1}^{5}\left[\left(\alpha_{i}\left(\lambda_{i}\right)^{-\beta_{i}}+\mu_{i} m c_{i}\right)\left(n_{i}+\exp \left(n_{i} / 4\right)\right)\right]
$$

\section{ALGORITHM 1}

\section{Pseudo-code of the implemented EF-COA.}

1. Input the parameters: $H, K, \Omega, \Phi_{j}, N_{\text {Iter }}$.

2. Generate random number of nests for each habitat according to Equation (4).

3. While $G \leq N_{\text {Iter }}$

4. Evaluate the system cost (each nest) according to Equation (5).

5. Constraint handling using Equation (6).

6. Identify the best solution (minimum system cost) and migration (save this best solution) according to Equation (7).

7. Use the saved solution to create new habitats and nests according to Equation (8).

9. End while

10. Display the minimum system cost and the optimal values. 
F I G U RE 1 Flowchart of the implemented efficient cuckoo optimization algorithm (EF-COA)

F I G URE 2 Parallel-series system

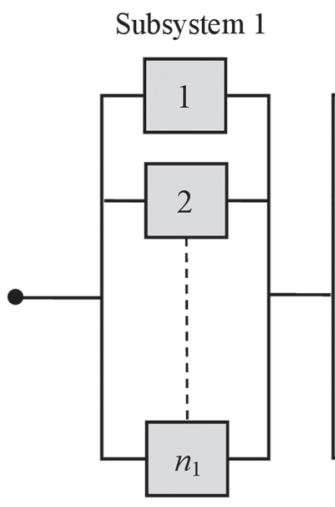

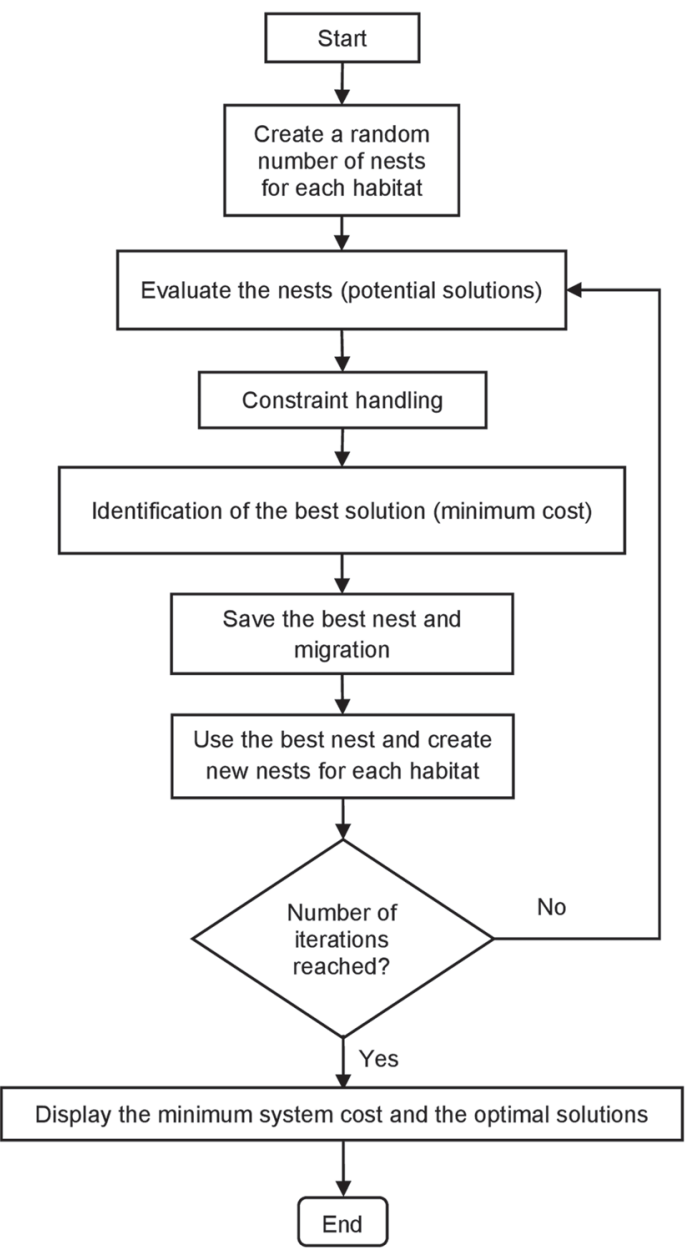

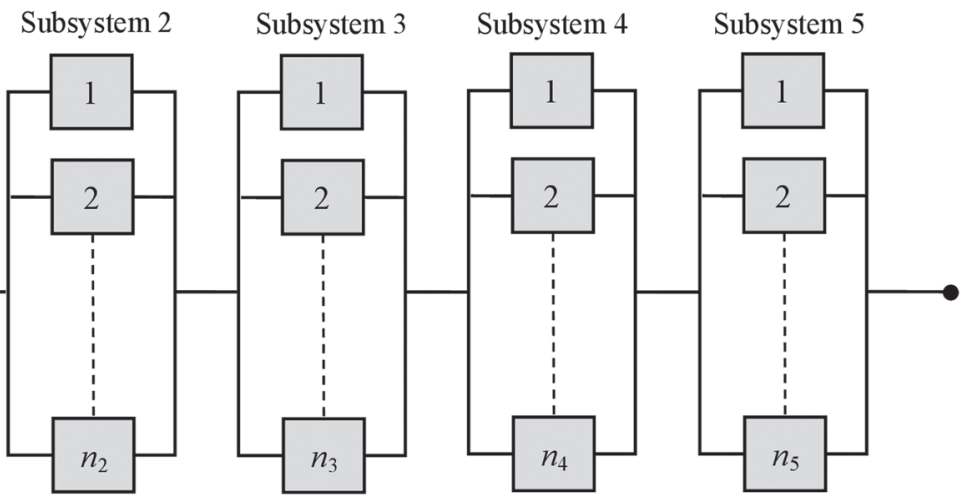

T A B L E 1 Data used in parallel-series and $n$-stage standby systems

\begin{tabular}{|c|c|c|c|c|c|}
\hline Subsystem $i$ & $\alpha_{i}\left(10^{-5}\right)$ & $\beta_{i}$ & $m c_{i}$ & $p_{i}$ & $w_{i}$ \\
\hline 2 & 1.45 & 1.5 & 5000 & 2 & 8 \\
\hline 4 & 8.05 & 1.5 & 5000 & 4 & 6 \\
\hline 5 & 1.95 & 1.5 & 5000 & 2 & 9 \\
\hline
\end{tabular}




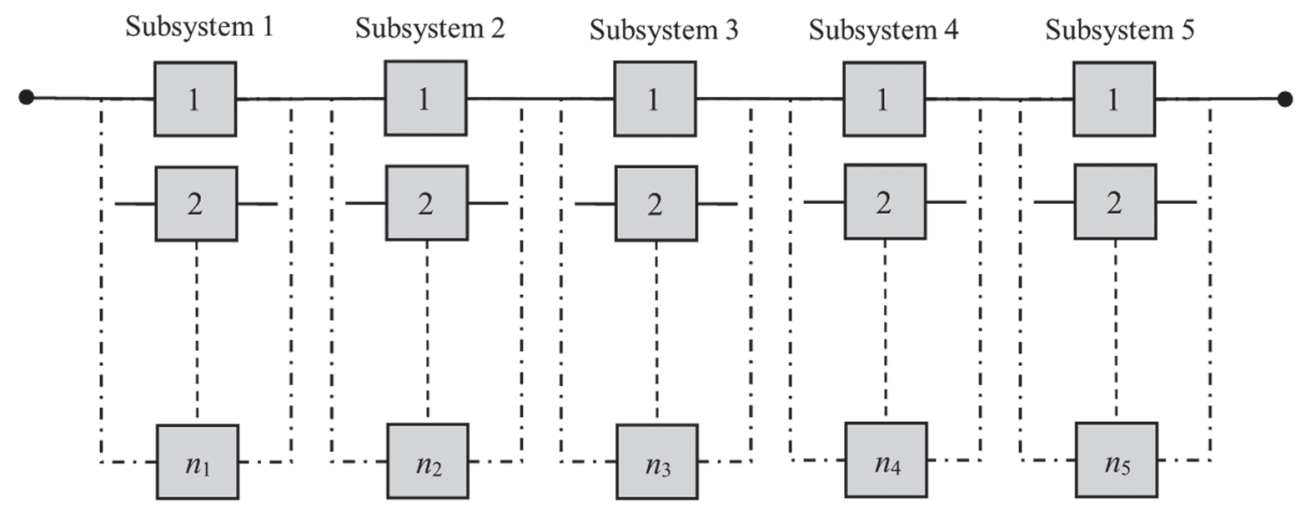

F I G U R E 3 n-stage standby system

Subject to

$$
\begin{gathered}
\sum_{i=1}^{5} p_{i}\left(n_{i}\right)^{2} \leq 150, \\
\sum_{i=1}^{5} w_{i} n_{i} \exp \left(n_{i} / 4\right) \leq 200, \\
\prod_{i=1}^{5}\left[1-\left(\sum_{k=0}^{n_{i}}\left(\frac{\lambda_{i}}{\mu_{i}}\right)^{k}\right)^{-1}\left(\frac{\lambda_{i}}{\mu_{i}}\right)^{n_{i}}\right] \geq 0.9, \\
n_{i} \geq 1 ; n_{i} \in \mathbb{Z}^{+} \\
\lambda_{i} \in\left[10^{-7}, 10^{-3}\right] \subset \mathfrak{R}^{+} \\
\mu_{i} \in\left[32 \times 10^{-7}, 32 \times 10^{-3}\right] \subset \mathfrak{R}^{+} \\
i=1,2, \ldots, 5 .
\end{gathered}
$$

The data and the constraints (14) and (15) are the same as for the parallel-series system. However, the system availability requirement constraint formulated in Equation (16) is more complex than that in Equation (12).

\section{5 | RESULTS AND DISCUSSION}

The developed EF-COA has been coded using the MATLAB programming language and run on a personal computer with a Processor G620 (2.60 GHz Sandy Bridge, 4 GB of RAM and 3 Mo of cache memory) under the Windows 7 to 64bits operating system. The number of habitats and the maximum number of nests per habitat is 10 . The number of iterations is fixed at 50, ie, the maximum number of function evaluations that the algorithm may use is 5000. The base COA has been also applied to compare the results.

Tables 2 and 3 report the results for the two case studies. The best values of the system cost and number of function evaluations (NFE) are highlighted in bold. In Table 2, the cost obtained by the EF-COA for the parallel-series system is 214.1934 (in arbitrary cost units), which is smaller than that of COA (214.2662) and TA-GA (214.7794). ${ }^{14}$ The EF-COA also used the lowest NFE (5000) compared with the other methods, 30000 and 40 000, respectively. From Table 3, it can be observed that the cost provided by the EF-COA (234.9172) for the $n$-stage standby system is less than the result of the TA-GA (236.8314) ${ }^{15}$ and the COA (236.2035). The NFE performance is also better, as for the parallel-series system. Furthermore, the standard deviations (SDs) of 20 independent runs reveal that the EF-COA is more stable than the COA, ie, smaller SD.

Figures 4-6 highlight the performances of the proposed EF-COA for the parallel-series system and the $n$-stage standby system, respectively. 
T A B L E 2 Results for the parallel-series system

\begin{tabular}{|c|c|c|c|c|c|c|c|c|}
\hline Method & $n$ & $\lambda\left(10^{-3}\right)$ & $\mu\left(10^{-2}\right)$ & $\boldsymbol{A}$ & $A_{s}$ & $C_{s}$ & NFE & SD \\
\hline TA-GA ${ }^{14}$ & $(3,2,2,3,3)$ & $\begin{array}{l}(0.3584,0.2236 \\
0.1447,0.6568 \\
0.3401)\end{array}$ & $\begin{array}{l}(0.10,0.14,0.09 \\
0.15,0.09)\end{array}$ & $\begin{array}{l}(0.9830,0.9802 \\
0.9812,0.9726 \\
0.9789)\end{array}$ & 0.9000 & 214.7794 & 40,000 & - \\
\hline $\mathrm{COA}$ & $(3,2,2,3,3)$ & $\begin{array}{l}(0.3640,0.2307 \\
0.1466,0.6469 \\
0.3419)\end{array}$ & $\begin{array}{l}(0.1018,0.1317 \\
0.0999,0.1449 \\
0.0965)\end{array}$ & $\begin{array}{l}(0.9817,0.9777 \\
0.9836,0.9705 \\
0.9820)\end{array}$ & 0.9000 & 214.2662 & 30,000 & $6.32 \mathrm{E}-03$ \\
\hline
\end{tabular}

Abbreviations: EF-COA, efficient cuckoo optimization algorithm; NFE, number of function evaluations; SD, standard deviation; TA-GA, Tabu search and genetic algorithm. Bold values represent the best results.

T A B L E 3 Results for the $n$-stage standby system

\begin{tabular}{|c|c|c|c|c|c|c|c|c|}
\hline Method & $n$ & $\lambda\left(10^{-3}\right)$ & $\mu\left(10^{-2}\right)$ & $\boldsymbol{A}$ & $A_{s}$ & $C_{s}$ & NFE & SD \\
\hline TA-GA ${ }^{15}$ & $(3,3,2,3,2)$ & $\begin{array}{l}(0.3261,0.2749 \\
0.1507,0.5963 \\
0.2555)\end{array}$ & $\begin{array}{l}(0.12,0.10,0.10 \\
0.17,0.15)\end{array}$ & $\begin{array}{l}(0.9852,0.9838 \\
0.9808,0.9710 \\
0.9750)\end{array}$ & 0.9000 & 236.8314 & 40,000 & - \\
\hline $\mathrm{COA}$ & $(2,3,2,3,3)$ & $\begin{array}{l}(0.2737,0.2712 \\
0.1382,0.5901 \\
0.3106)\end{array}$ & $\begin{array}{l}(0.1548,0.0970 \\
0.0998,0.1685 \\
0.1068)\end{array}$ & $\begin{array}{l}(0.9741,0.9841 \\
0.9834,0.9716 \\
0.9824)\end{array}$ & 0.9000 & 236.2035 & 30,000 & $5.17 \mathrm{E}-02$ \\
\hline
\end{tabular}

Abbreviations: EF-COA, efficient cuckoo optimization algorithm; NFE, number of function evaluations; SD, standard deviation; TA-GA, Tabu search and genetic algorithm. Bold values represent the best results.

F I G URE 4 System cost for the parallel-series system [Colour figure can be viewed at wileyonlinelibrary.com]

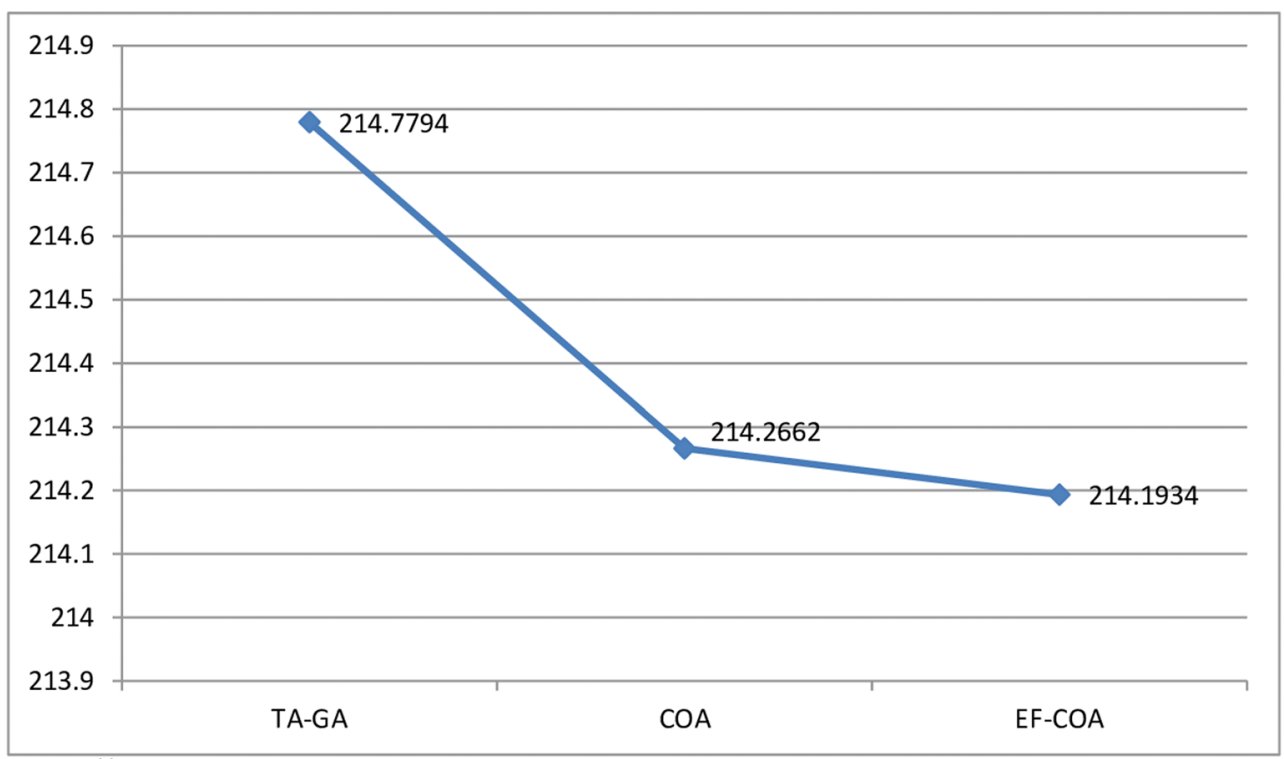




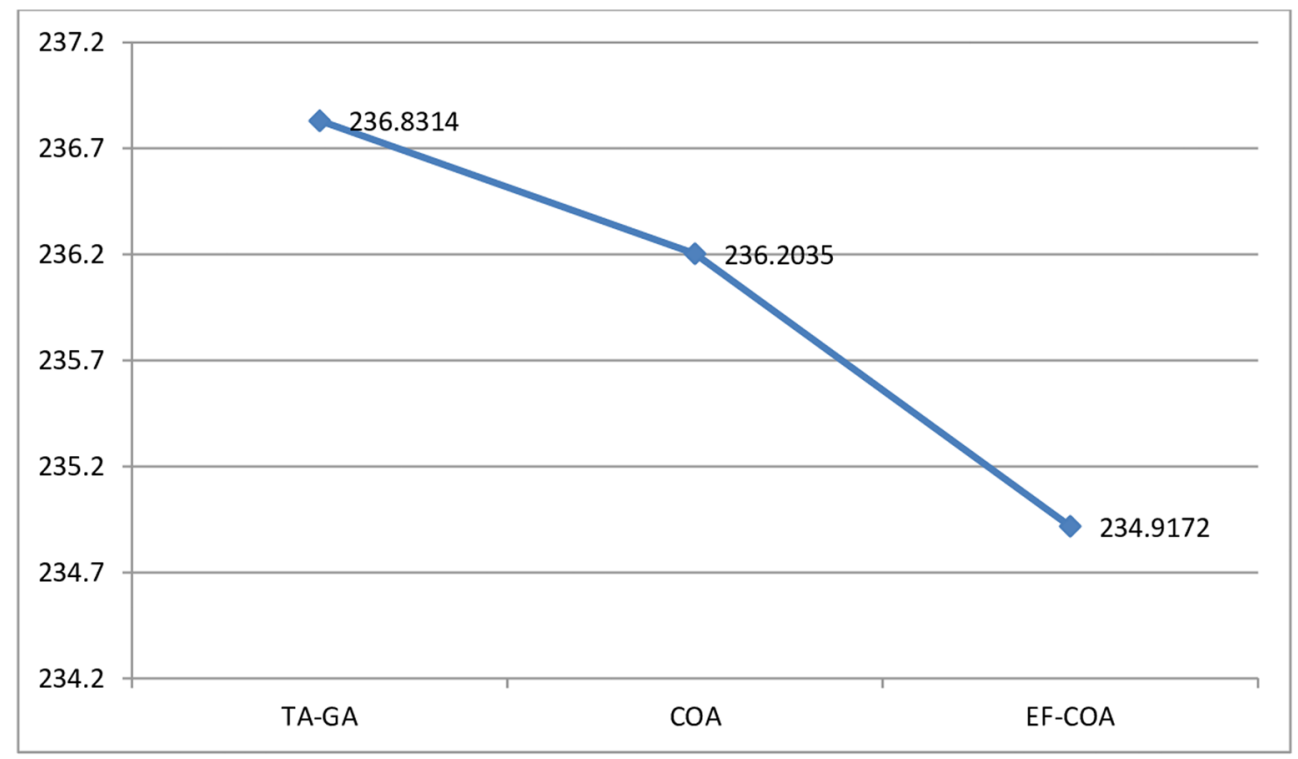

TA-GA ${ }^{15}$

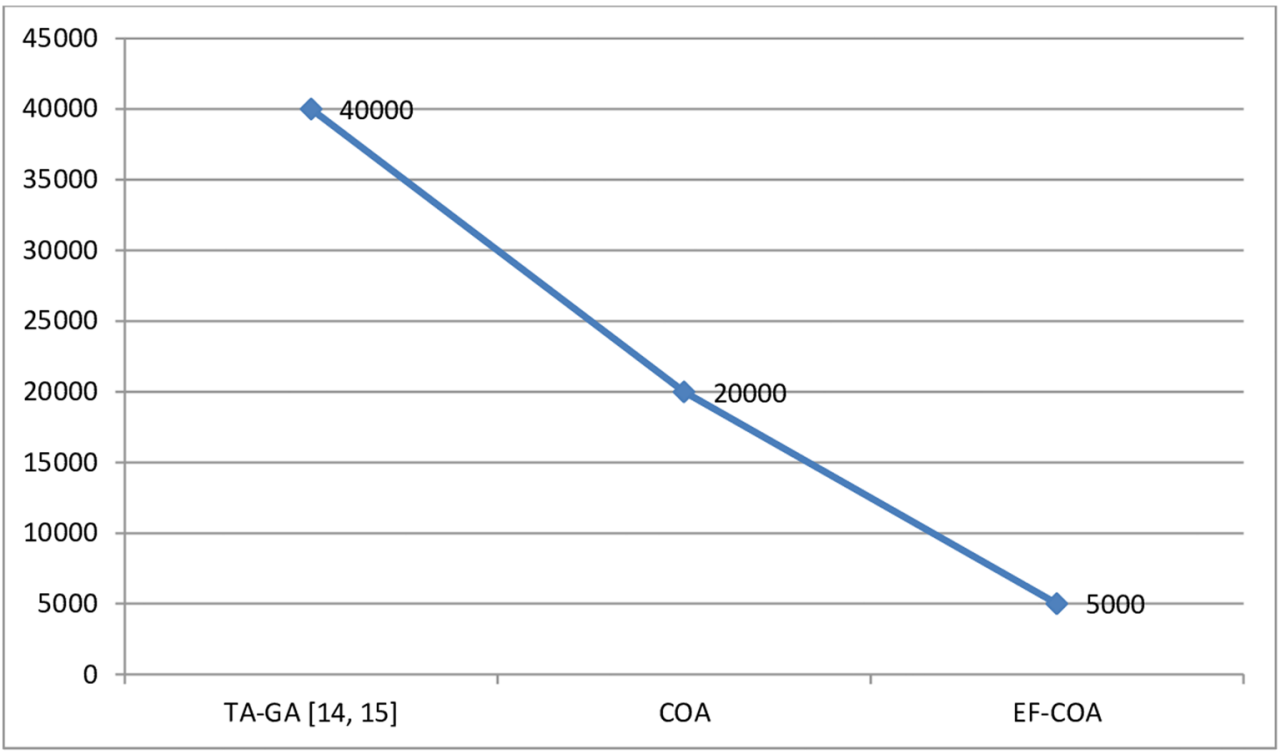

TA-GA ${ }^{14,15}$
F I G URE 5 System cost for the $n$-stage standby system

[Colour figure can be viewed at wileyonlinelibrary.com]
FIG URE 6 Number of function evaluations (NFE) for the parallel-series system and the $n$-stage standby system [Colour figure can be viewed at wileyonlinelibrary.com]

\section{6 | CONCLUSIONS}

In this paper, a new solution approach for minimizing the system cost of repairable systems subject to availability constraints has been proposed. A novel method based on the habitats and floating nests of the cuckoo, called the EF-COA, has been developed. The standard COA uses the egg-laying radius (ELR), which may slow down the algorithm when solving a complex problem. In the EF-COA, various habitats consisting of different nests are implemented in order to improve the quality on the solution and the performances. Therefore, the ELR has been avoided, while the system cost has been modeled as a nest. Application to two numerical case studies, ie, parallel-series system and $n$-stage standby system, has demonstrated the effectiveness of the proposed method in terms of better solutions and fewer function evaluations. Future research efforts will be devoted to extending the method for treating and addressing multi-objective optimization problems and a comprehensive industrial case study. 


\section{NOMENCLATURE}

$A \quad=\left(A_{1}, A_{2}, \ldots, A_{m}\right)$, vector of component availabilities for the system

$A_{i} \quad$ availability of each component in subsystem $i, 1 \leq i \leq m$

$A_{S} \quad$ system availability

$A_{S}^{*} \quad$ system availability requirement

$b \quad$ vector of resource limitation

$g_{j} \quad j$ th constraint function, $j=1, \ldots, M$

$m$ number of subsystems in the system

M number of constraints

$n=\left(n_{1}, n_{2}, \ldots, n_{m}\right)$, vector of redundancy allocation for the system

$n_{i} \quad$ number of components in subsystem $i, 1 \leq i \leq m$

$P \quad$ limitation on product of weight and volume

$R_{i} \quad=1-\left(1-r_{i}\right)^{n_{i}}$, reliability of the $i$ th subsystem, $1 \leq i \leq m$

$T \quad$ operating time during which the component must not fail (mission time, $T=1000$ )

$w_{i} \quad$ weight of each component in subsystem $i, 1 \leq i \leq m$

$\beta_{i}, \alpha_{i} \quad$ parameters representing physical features (shaping and scaling factors, respectively) of each component at subsystem $i, 1 \leq i \leq m$

$\mu \quad=\left(\mu_{1}, \mu_{2}, \ldots, \mu_{m}\right)$, vector of component repair rates for the system

$\mu_{i} \quad$ repair rate of each component in subsystem $i, 1 \leq i \leq m$

$\lambda \quad=\left(\lambda_{1}, \lambda_{2}, \ldots, \lambda_{m}\right)$, vector of component failure rates for the system

$\lambda_{i} \quad$ failure rate of each component in subsystem $i, 1 \leq i \leq m$

$\lambda_{i L}, \mu_{i L} \quad$ lower limits on the failure rate and repair rate of each component in subsystem $i, 1 \leq i \leq m$

$\lambda_{i U}, \mu_{i U}$ upper limits on the failure rate and repair rate of each component in subsystem $i, 1 \leq i \leq m$

\section{ORCID}

Mohamed Arezki Mellal (D) https://orcid.org/0000-0003-0667-8851

\section{REFERENCES}

1. Marseguerra M, Zio E, Martorell S. Basics of genetic algorithms optimization for RAMS applications. Reliab Eng Syst Saf. 2006;91: 977-991.

2. Marseguerra M, Zio E, Podofillini L. Optimal reliability/availability of uncertain systems via multi-objective genetic algorithms. IEEE Trans Reliab. 2004;53:424-434.

3. Lin YH, Li YF, Zio E. Fuzzy reliability assessment of systems with multiple-dependent competing degradation processes. IEEE Trans Fuzzy Syst. 2015;23:1428-1438. https://doi.org/10.1109/TFUZZ.2014.2362145

4. Zio E. Reliability engineering: old problems and new challenges. Reliab Eng Syst Saf. 2009;94:125-141.

5. Giuggioli Busacca P, Marseguerra M, Zio E. Multiobjective optimization by genetic algorithms: application to safety systems. Reliab Eng Syst Saf. 2001;72:59-74.

6. Garg H, Rani M, Sharma SP. An efficient two phase approach for solving reliability-redundancy allocation problem using artificial bee colony technique. Comput Oper Res. 2013;40:2961-2969. https://doi.org/10.1016/j.cor.2013.07.014

7. Hsieh YC, You PS. An effective immune based two-phase approach for the optimal reliability-redundancy allocation problem. Appl Math Comput. 2011;218:1297-1307. https://doi.org/10.1016/j.amc.2011.06.012

8. Liu Y, Qin G. A DE algorithm combined with Lévy flight for reliability redundancy allocation problems. Int J Hybrid Inf Technol. 2015;8: 113-118.

9. Garg H. An efficient biogeography based optimization algorithm for solving reliability optimization problems. Swarm Evol Comput. 2015;24:1-10. https://doi.org/10.1016/j.swevo.2015.05.001

10. Zhang E, Chen Q. Multi-objective reliability redundancy allocation in an interval environment using particle swarm optimization. Reliab Eng Syst Saf. 2016;145:83-92. https://doi.org/10.1016/j.ress.2015.09.008

11. Lins ID, Moura MC, Zio E, Droguett EL. A particle swarm-optimized support vector machine for reliability prediction. Qual Reliab Eng Int. 2012;28:141-158.

12. Mellal MA, Zio E. A penalty guided stochastic fractal search approach for system reliability optimization. Reliab Eng Syst Saf. 2016;152: 213-227.

13. Kumar A, Pant S, Ram M. System reliability optimization using gray wolf optimizer algorithm. Qual Reliab Eng Int. 2016;33(7): 1327-1335.

14. Liu GS. Availability optimization for repairable parallel-series system by applying Tabu-GA combination method. In: 10th IEEE International Conference on Industrial Informatics. Beijin, China. 2012: 803-808 
15. Liu GS. Availability optimization for repairable n-stage standby system by applying Tabu-GA combination method. Int J Model Optim. 2013;3:245-250.

16. Shelokar PS, Jayaraman VK, Kulkarni BD. Ant algorithm for single and multiobjective reliability optimization problems. Qual Reliab Eng Int. 2002;18:497-514. https://doi.org/10.1002/qre.499

17. Mellal MA, Zio E. System reliability-redundancy allocation by evolutionary computation. In: 2nd International Conference on System Reliability and Safety. IEEE, Milan, Italy 2017

18. Cha JW, Finkelstein M. On preventive maintenance under different assumptions on the failure/repair processes. Qual Reliab Eng Int. 2018;34:66-77.

19. Yu J, Zheng S, Pham H, Chen T. Reliability modeling of multi-state degraded repairable systems and its applications to automotive systems. Qual Reliab Eng Int. 2018;34(3):459-474. https://doi.org/10.1002/qre.2265

20. Rajabioun R. Cuckoo optimization algorithm. Appl Soft Comput. 2011;11:5508-5518.

21. Mellal MA, Adjerid S, Williams EJ, Benazzouz D. Optimal replacement policy for obsolete components using cuckoo optimization algorithm based-approach: dependability context. J Sci Ind Res (India). 2012;71:715-721.

22. Mellal MA, Adjerid S, Williams EJ. Optimal selection of obsolete tools in manufacturing systems using cuckoo optimization algorithm. Chem Eng Trans. 2013;33:355-360. https://doi.org/10.3303/CET1333060

23. Amiri E, Mahmoudi S. Efficient protocol for data clustering by fuzzy cuckoo optimization algorithm. Appl Soft Comput. 2016;41:15-21.

24. Mellal MA, Williams EJ. Parameter optimization of advanced machining processes using cuckoo optimization algorithm and hoopoe heuristic. J Intell Manuf. 2016;27:927-942.

25. Mellal MA, Williams EJ. Cuckoo optimization algorithm for unit production cost in multi-pass turning operations. Int J Adv Manuf Technol. 2015;76:647-656. https://doi.org/10.1007/s00170-014-6309-2

26. Mellal MA, Williams EJ. Total production time minimization of a multi-pass milling process via cuckoo optimization algorithm. Int $J$ Adv Manuf Technol. 2016;87(1-4):747-754. https://doi.org/10.1007/s00170-016-8498-3

27. Rabiee M, Sajedi H. Job scheduling in grid computing with cuckoo optimization algorithm. Int J Comput Appl. 2013;62:38-44.

28. Roozitalab A, Asgharizadeh E. Optimizing the warranty period by cuckoo meta-heuristic algorithm in heterogeneous customers' population. J Ind Eng Int. 2013;9:1-6.

29. Mellal MA, Williams EJ. Cuckoo optimization algorithm with penalty function for combined heat and power economic dispatch problem. Energy. 2015;93:1711-1718. https://doi.org/10.1016/j.energy.2015.10.006

30. Khormali A, Addeh J. A novel approach for recognition of control chart patterns: type-2 fuzzy clustering optimized support vector machine. ISA Trans. 2016;63:256-264. https://doi.org/10.1016/j.isatra.2016.03.004

31. Deb K. An efficient constraint handling method for genetic algorithms. Comput Methods Appl Mech Eng. 2000;186:311-338. https://doi. org/10.1016/S0045-7825(99)00389-8

32. Subbaraj P, Rengaraj R, Salivahanan S. Enhancement of combined heat and power economic dispatch using self adaptive real-coded genetic algorithm. Appl Energy. 2009;86:915-921. https://doi.org/10.1016/j.apenergy.2008.10.002

\section{AUTHOR BIOGRAPHIES}

Mohamed Arezki Mellal has a PhD in mechatronics. He is an Associate Professor (with accreditation to supervise research) at the Department of Mechanical Engineering, Faculty of Technology, M'Hamed Bougara University, Algeria and a Visiting Scholar at the Center for Advanced Life Cycle Engineering, Department of Mechanical Engineering, University of Maryland, College Park, MD, USA. Likewise, he was a Visiting Scholar at Osaka ElectroCommunication University, Japan; Korea Aerospace University, South Korea; and University of Essex, UK. He is a member of the Algerian National Laboratory for Maintenance Education in conjunction with the European Union (Erasmus+). He has published in several journal and conference proceedings. He has edited three books and authored four book chapters. Dr Mellal was an Invited Keynote Lecturer at the plenary sessions of the Day of Science 2015, Boumerdes, Algeria and of the International Symposium on Technology and Sustainable Industry Development, ISTSID 2019, El-Oued, Algeria. He was a Session Chair for the 2nd and 3rd International Conference on System Reliability and Safety, ICSRS 2017 and 2018, Milan (Italy) and Barcelona (Spain), respectively. He has been selected as a Best Reviewer for the International Conference on Vision, Image and Signal Processing, ICVISP 2017 and 2018, Osaka (Japan) and Las Vegas (USA), respectively. He has also been a committee member for over seventy international conferences. He serves as a regular reviewer for $18 \mathrm{SCI}$-indexed journals and an editorial board member in seven peer-reviewed international journals.

Enrico Zio received the MSc degree in nuclear engineering from the Politecnico di Milano, Milan, Italy, in 1991, the MSc degree in mechanical engineering from the University of California at Los Angeles, Los Angeles, CA, USA, in 1995, the PhD degree in nuclear engineering from the Politecnico di Milano, in 1996, and the PhD degree in probabilistic risk assessment from the Massachusetts Institute of Technology (MIT), Cambridge, MA, USA, in 1998. He 
is currently a Full Professor with the Centre for Research on Risk and Crises, Ecole de Mines, ParisTech, PSL University, Sophia Antipolis, France, a Full Professor and the President of the Alumni Association at Politecnico di Milano, an Eminent Scholar with Kyung Hee University, Seoul, South Korea, a Distinguished Guest Professor with Tsinghua University, Beijing, China, an Adjunct Professor with the City University of Hong Kong, Hong Kong, Beihang University, Beijing, and Wuhan University, Wuhan, China, and the Co-Director of the Center for Reliability and Safety of Critical Infrastructures and the SinoFrench Laboratory of Risk Science and Engineering, Beihang University. He has authored or coauthored over seven books and 300 papers on international journals. His current research interests include modeling of the failure-repair-maintenance behavior of components and complex systems, analysis of their reliability, maintainability, prognostics, safety, vulnerability, resilience, and security characteristics, and development and use of the Monte Carlo simulation methods, artificial techniques, and optimization heuristics. Dr Zio is the Chairman and Co-Chairman of several international conferences, an Associate Editor of several international journals, and a referee of more than 20 .

Edward J. Williams holds bachelor's and master's degrees in Mathematics (Michigan State University, 1967; University of Wisconsin, 1968). From 1969 to 1971, he did statistical programming and analysis of biomedical data at Walter Reed Army Hospital, Washington, D.C. He joined Ford Motor Company in 1972 where he worked until retirement in December 2001 as a computer software analyst supporting statistical and simulation software. After retirement from Ford, he joined PMC, Dearborn, Michigan, as a senior simulation analyst. Also, since 1980, he has taught classes at the University of Michigan, including both undergraduate and graduate simulation classes using GPSS/H, SLAM II, SIMAN, ProModel, SIMUL8, or Arena. He is a member of the Institute of Industrial Engineers (IIE), the Society for Computer Simulation International (SCS), and the Michigan Simulation Users Group (MSUG). He serves on the editorial board of the International Journal of Industrial Engineering-Applications and Practice. During the last several years, he has given invited plenary addresses on simulation and statistics at conferences in Monterrey, México; İstanbul, Turkey; Genova, Italy; Rīga, Latvia; and Jyväskylä, Finland. He served as a coeditor of Proceedings of the International Workshop on Harbour, Maritime and Multimodal Logistics Modelling and Simulation 2003, a conference held in Rīga, Latvia. Likewise, he served the Summer Computer Simulation Conferences of 2004, 2005, and 2006 as Proceedings coeditor. He was the Simulation Applications track coordinator for the 2011 Winter Simulation Conference. A paper he coauthored with three of his simulation students won "best paper in track" award at the Fifth International Conference on Industrial Engineering and Operations Management, held in Dubai, United Arab Emirates, in March 2015.

How to cite this article: Mellal MA, Zio E, Williams EJ. Cost minimization of repairable systems subject to availability constraints using efficient cuckoo optimization algorithm. Qual Reliab Engng Int. 2020;36:1098-1110. https://doi.org/10.1002/qre.2617 


\section{APPENDIX A.}

Step 1. Generate initial cuckoo habitat.

The initial set of solutions represents the cuckoo habitat as follows:

$$
\text { Habitat }=\left[x_{1}, x_{2}, \ldots, x_{N_{\mathrm{var}}}\right] \text {, }
$$

where $X$ is the vector of the solutions and $N_{v a r}$ is the number of variables in the problem. A matrix of size $N_{\text {pop }} \times N_{v a r}$ is generated.

Step 2. Evaluation.

The fitness of each line is evaluated, where $N_{p o p}$ is the number of lines. The habitat is evaluated as a fitness function.

Step 3. Egg allocation.

Dedicate some eggs to each cuckoo.

Step 4. Egg-laying radius.

The cuckoos start to lay eggs in the area according to a distance called egg-laying radius (ELR), as follows:

$$
\text { ELR }=\alpha \times \frac{\text { Number of current cuckoo's eggs }}{\text { Total number of eggs }} \times\left(\operatorname{var}_{h i}-\operatorname{var}_{l o w}\right),
$$

where $\alpha$ is an integer and $v a r_{h i}$ and $v a r_{l o w}$ are the upper and lower bounds of the variables. Some eggs will be destroyed by the host birds and $10 \%$ of the survival cuckoos will starve.

Step 5. Migration.

When the cuckoos become mature, the cuckoos' swarm will migrate to achieve the best goal. The different groups are classified using the $K$-means clustering. Each cuckoo fly $U \%$ of all the way toward destination (where $U$ is a random number uniformly distributed between 0 and 1$)$, with a deviation $\varphi(\Pi / 6 \mathrm{rad})$.

Step 6. Population limit.

A maximum number of cuckoos is considered to limit the population.

Step 7. Repeat steps 2 to 6 until the stopping condition is satisfied. 